\title{
Limiting and controlling carbapenem-resistant Klebsiella pneumoniae
}

\author{
This article was published in the following Dove Press journal: \\ Infection and Drug Resistance \\ 9 December 2013 \\ Number of times this article has been viewed
}

\author{
Lisa Saidel-Odes ${ }^{1,2}$ \\ Abraham Borer ${ }^{1,2}$ \\ 'Infection Control and Hospital \\ Epidemiology Unit, ${ }^{2}$ Infectious \\ Diseases Institute, Soroka University \\ Medical Center and the Faculty \\ of Health Sciences, Ben-Gurion \\ University of the Negev, \\ Beer-Sheva, Israel
}

\begin{abstract}
Carbapenem-resistant Klebsiella pneumoniae (CRKP) is resistant to almost all antimicrobial agents, is associated with substantial morbidity and mortality, and poses a serious threat to public health. The ongoing worldwide spread of this pathogen emphasizes the need for immediate intervention. This article reviews the global spread and risk factors for CRKP colonization/infection, and provides an overview of the strategy to combat CRKP dissemination.
\end{abstract}

Keywords: carbapenem-resistant Klebsiella pneumoniae, infection control, cohort, active surveillance, rectal cultures

\section{Introduction}

During the last decade, carbapenem-resistant Klebsiella pneumoniae (CRKP) has spread throughout the world, becoming a matter of great concern. CRKP was first reported in the United States in 2001 in North Carolina. ${ }^{1}$ The first case outside the United States occurred in France, where a patient who had been hospitalized in New York carried the strain with him. ${ }^{2}$ Since then, CRKP has been reported in Europe, ${ }^{3-8}$ the Middle East, ${ }^{9-12}$ South America, ${ }^{13,14}$ and the Far East. ${ }^{15,16}$ Several outbreaks of CRKP have occurred around the world as described in Table 1.3,4,9-11,13,14,16-18 These outbreaks have been associated with the plasmid-encoded carbapenemase $K$. pneumoniae carbapenemase (KPC), a carbapenem-hydrolyzing $\beta$-lactamase. ${ }^{19}$ CRKP isolates are resistant to almost all available antimicrobials. These isolates are susceptible only to polymyxins and tigecycline; a minority are also susceptible to the few remaining aminoglycosides, though resistance to these agents is worrisomely increasingly reported..$^{20,21}$

K. pneumoniae, an inhabitant of the gastrointestinal tract, skin, and nasopharynx, can cause infection in many parts of the body, including urinary tract infections, hospital acquired pneumonia, intra-abdominal infections, wound infections, and primary bacteremia. ${ }^{22-24}$ Initially, CRKP seemed to be limited to causing hospital acquired infections, though further on, CRKP has spread in different health care systems, including long-term care facilities. ${ }^{25-29}$ The mortality rate of CRKP infections (mainly bacteremia) seems strikingly high, $30 \%-50 \% .^{17,30-32}$

\section{Predictors for CRKP colonization}

Several investigators have evaluated predictors for CRKP colonization. The following summarizes various studies.

- Poor functional status, intensive care unit (ICU) stay, and the recipient of antibiotics were predictors of CRKP colonization in a study by Schwaber et al. ${ }^{31}$ Exposure to fluoroquinolones was independently predictive of CRKP isolation.
Infectious Diseases Institute Soroka University Medical Center, PO Box I5I, Beer-Sheva 84I0I, Israel Tel +97286400370

Fax +97286403534

Email saidelod@bgu.ac.il 
Table I Outbreaks of CRKP described around the globe

\begin{tabular}{|c|c|c|c|c|}
\hline Reference & Country & $\begin{array}{l}\text { Year of } \\
\text { outbreak }\end{array}$ & $\begin{array}{l}\text { Number } \\
\text { of cases }\end{array}$ & Intervention \\
\hline Samra et al $^{9}$ & Israel & 2006 & 90 & $\begin{array}{l}\text { Active surveillance for CRKP at a national level was suggested } \\
\text { (implemented from February 2007). }\end{array}$ \\
\hline Zhang et $\mathrm{al}^{16}$ & $\begin{array}{l}\text { People's Republic } \\
\text { of China }\end{array}$ & 2007 & 28 & Not available. \\
\hline Souli et al ${ }^{17}$ & Greece & $2007-2008$ & 50 & $\begin{array}{l}\text { Point prevalence survey of environmental colonization of CRKP } \\
\text { conducted in ICU. } \\
\text { Infection control measures were intensified throughout the hospital. } \\
\text { Dedicated infection control personnel to ensure hand hygiene, contact } \\
\text { isolation, environmental cleaning. } \\
\text { Cohorting of colonized or infected patients when possible. } \\
\text { Carbapenem restriction enforced. }\end{array}$ \\
\hline Gregory et al ${ }^{13}$ & Puerto Rico & 2008 & 26 & Active surveillance for CRKP colonization, cohorting of CRKP patients. \\
\hline Lopez et al ${ }^{14}$ & Colombia & 2008 & 84 & $\begin{array}{l}\text { Investigation for source patient. } \\
\text { Isolation, barrier precautions, surveillance cultures for all ICU patients. }\end{array}$ \\
\hline $\begin{array}{l}\text { Wiener-Well } \\
\text { et al }{ }^{10}\end{array}$ & Israel & 2009 & 16 & $\begin{array}{l}\text { Point prevalence survey. } \\
\text { Oral, perianal, and rectal swabs obtained from all hospitalized patients. }\end{array}$ \\
\hline $\begin{array}{l}\text { Carbonne } \\
\text { et } \mathrm{al}^{18}\end{array}$ & France & 2009 & 13 & $\begin{array}{l}\text { Limiting transfer of cases and contact patients to other wards. } \\
\text { Cohorting separately cases and contact patients. } \\
\text { Reinforcing hand hygiene and contact precautions. } \\
\text { Systematic screening of contact patients. }\end{array}$ \\
\hline $\begin{array}{l}\text { Robustillo } \\
\text { Rodela et } \text { al }^{3}\end{array}$ & Spain & 2009 & 7 & $\begin{array}{l}\text { Stool surveillance cultures from patients and staff. } \\
\text { Hand swabs taken from staff and patients' relatives. } \\
\text { Environmental samples. } \\
\text { Patients placed in single rooms under contact precautions and daily } \\
\text { washing with } 4 \% \text { chlorhexidine soap. } \\
\text { Alcohol-based disinfectant used on surfaces. }\end{array}$ \\
\hline $\begin{array}{l}\text { Steinmann } \\
\text { et } \mathrm{al}^{4}\end{array}$ & Germany & 2010 & 7 & $\begin{array}{l}\text { Cases were isolated in single-bedrooms with strict contact precautions. } \\
\text { Barrier nursing. } \\
\text { Contact precautions also for patients who shared a room with a case. } \\
\text { Weekly surveillance cultures from all ICU patients and for patients who } \\
\text { moved wards during hospitalization. } \\
\text { Environmental investigation. }\end{array}$ \\
\hline Balkhy et al" & Saudi Arabia & 2010 & 20 & $\begin{array}{l}\text { Strict contact isolation, intensifying hand hygiene compliance, CRKP } \\
\text { active surveillance, staff education. }\end{array}$ \\
\hline
\end{tabular}

Abbreviations: CRKP, carbapenem-resistant Klebsiella pneumoniae; ICU, intensive care unit.

- Nursing home residency before hospital admission, bedridden status, and previous antibiotic therapy were demonstrated to predict CRKP colonization in a study by Borer et al. ${ }^{33}$

- Previous use of carbapenem and cephalosporin were found to predict CRKP colonization in a study by Kwak et al. ${ }^{34}$ Nosocomial isolation of CRKP was strongly favored by the selection pressure of carbapenem. In this study, prior treatment with fluoroquinolones was associated with decreased risk for the emergence of CRKP.

\section{Risk factors for CRKP infection}

Risk factors for acquisition of CRKP infection by patients initially colonized with CRKP have been researched, results are listed below.

- Diabetes mellitus, solid tumors, previous invasive procedures, tracheostomy, urinary catheter insertion, and antipseudomonal penicillin therapy were risk factors for the acquisition of CRKP infection in a study by Borer et al. ${ }^{33}$ Carbapenem use was strongly predictive of CRKP infection.

- ICU admission (within 2 weeks) or prior exposure to carbapenems or glycopeptides were independent risk factors for the acquisition of nosocomial CRKP infections in a study by Wu et al. ${ }^{35}$

- Antibiotic use, especially colistin, presence of a urinary catheter, surgery, invasive procedures, and ICU admission were risk factors for the acquisition of CRKP infection in a study by Shilo et al. ${ }^{22}$

- In a multivariate analysis, prior use of macrolides and any antibiotic exposure $\geq 14$ days remained the only independent factors associated with CRKP bacteremia in a study by Hussein et al. ${ }^{36}$ In a univariate analysis, CRKP bacteremia was associated with hematological 
malignancy, chronic renal failure, chronic liver disease, previous bone marrow transplantation, longer length of stay before the onset of bacteremia, receipt of mechanical ventilation, central venous catheterization, dialysis, and stay in the ICU or hematology department. ${ }^{36}$

- Independent predictors of subsequent carbapenem-resistant Enterobacteriaceae (CRE) infection were admission to the ICU, having a central venous catheter, receipt of antibiotics, and diabetes mellitus in a study by Schechner et al. ${ }^{37}$

\section{Persistent CRKP colonization}

Persistent gastrointestinal carriage of CRKP is associated with (any) catheter use and a low functional status. It is more common in patients with recent CRKP acquisition ( $\leq 4$ months) and related to residence in a long-term care facility. ${ }^{38}$ Multiple hospitalizations and CRKP disease extend the duration of carriage, with colonization detected after 12 months in $39 \%$ of carriers. ${ }^{39}$

Dissemination of CRKP represents a serious threat to public health. ${ }^{40,41}$ The fact that CRKP strains have spread worldwide, the lack of proven clinically efficacious antibiotics, and the presence of gene-encoded KPC enzymes residing on transmissible plasmids makes the control of CRKP infections an imperative task of health care systems. ${ }^{42}$

The Centers for Disease Control and Prevention, as well as a meeting of national experts in Europe, have published new infection control recommendations and strategies to combat this public health concern. ${ }^{40,43}$ We aim to present a multifaceted intervention strategy to limit and control CRKP spread in health care systems based on these guidelines, the Israeli nationwide intervention, our personal experience, and the current literature.

\section{Strategy for preventing CRKP dissemination in hospitals}

We suggest applying a comprehensive infection control intervention in acute care hospitals, comprising of bundles of infection control measures:

1. Flagging system

a. Active surveillance culture on hospital admission

b. Pre-emptive contact isolation pending cultures

2. Building of cohort space or ward for CRKP positive patients

3. Intensive active surveillance in high-risk wards

4. Epidemiological investigations

5. Staff education

6. New antibiotic restriction policy
7. Addressing risk factors

8. Other interventions.

In addition, a dedicated full-time infection control practitioner is needed to handle the collection and daily analysis of data.

\section{Flagging system}

On entering the emergency room, a clerk identifies high-risk patients (prior to hospital admission) and places an identification mark (ie, a red sticker) on the patient's chart. High-risk patients for CRKP colonization include patients transferred from other hospitals, patients who were hospitalized in other hospitals in the past 6 months, and patients residing in long-term care facilities. Risk factors are then verified by nurses who sign the verification stamp. Patients known to be colonized with CRKP within the past 12 months, who do not have a negative rectal polymerase chain reaction (PCR), are admitted to the cohort.

\section{Active surveillance culture on ward admission}

Rectal cultures using rectal swabs are performed for all high-risk patients on ward admission by the ward's nurse. Cultures are sent directly to the bacteriology laboratory for prompt CRKP identification.

\section{Pre-emptive contact isolation pending cultures}

All high-risk patients are placed in pre-emptive isolation in the wards pending admission surveillance culture results. Strict contact precautions are applied (ie, use of gown and gloves by health care workers); signage is applied on the entrance to the high-risk patient's room; 1:4 ratio of trained nurses to patients; a dedicated housekeeping team. Patients with a positive rectal culture for CRKP are transferred to a cohort area for maximal contact isolation. Patients with a negative rectal culture for CRKP remain in the ward and are treated using standard precautions.

\section{Building of cohort space or ward for CRKP positive patients}

Cohort space should be designed as a separate ward or as part of an existing ward, sectioned off with clear signage. The number of beds should address the expected number of patients. An exclusively dedicated nursing staff should be assigned. The cohort is supplied with its own equipment, including an X-ray machine, electrocardiogram machine, and monitors. Disposable stethoscope, blood pressure cuff, and thermometers should also be supplied. The physician in 
charge is not fully dedicated to the cohort, but complies with strict contact precautions. Strict patient isolation is applied by the dedicated nursing personnel. A dedicated housekeeping team is trained for cleaning and disinfecting CRKP positive patients' units during hospitalization and after discharge.

Environmental disinfection using 2,000 ppm chlorine for 10 minutes is done twice daily; 70\% alcohol is used for computers and monitors. Patients undergo daily whole-body disinfection with antiseptics (chlorhexidine); this method has been proven efficacious in preventing bloodstream infections. ${ }^{44,45}$

Visitors require patient permission and are educated about hand hygiene, use of gowns, gloves, etc. It is best to limit visitors' number to a minimum. Immune suppressed people and children are not allowed to enter the cohort space.

All hospitalized patients colonized/infected with CRKP are transferred to this ward if their medical condition allows (providing the cohort ward can continue the treatment they received up to this point).

\section{Intensive active surveillance in high-risk wards}

High-risk wards identified by CRKP incidence are targeted for interventions, such as ICUs and step-down units. ${ }^{46-48}$ In our hospital, neurology and geriatric wards were also included. ${ }^{42}$ All patients in these wards undergo rectal cultures for CRKP detection on admission and once weekly until they are discharged (to detect in-hospital transmission). Patients with a rectal culture positive for CRKP should be transferred to a cohort area for maximal contact isolation.

\section{Epidemiologic investigations}

Identification of new CRKP colonized/infected patients within the hospital prompt an immediate and intensive review of case and contacts by the infectious control practitioner, enforcement of compliance with hand hygiene, contact precautions, and disinfectant protocols. Rectal cultures from patients who shared a room with the case are performed due to the possibility of cross-infection between roommates. The timely identification of colonized and/or infected patients is critical to the success of interrupting cross-transmission of CRE in health care facilities. ${ }^{23}$

In the case of an outbreak or clusters, cultures of health care workers' hands and environmental cultures are performed at the discretion of the hospital epidemiologist.

\section{Staff education}

Physicians, staff nurses, nursing aids, and housekeeping teams all receive training on the relevance of CRKP and its route of transmission. ${ }^{49}$ Familiarizing health care personnel with proper hand hygiene technique as well as its rationale is very important. ${ }^{40}$ Pamphlets explaining hand hygiene are placed in relevant areas for staff and family. Hand hygiene adherence is monitored and feedback is provided, ensuring maximal compliance. Hand hygiene stations are readily accessible (clean sinks and/or alcohol-based hand rubs). ${ }^{40}$ Revision and strict adherence to contact precautions is of paramount importance; this should also be monitored.

\section{Antibiotic restrictive policy}

We advise restriction of carbapenem use by infectious diseases specialists, using rigorous policy enforcement where approval of empiric carbapenem use requires the agreement of two infectious diseases physicians. We have also limited the number of carbapenems for use; meropenem is the only agent of this class available in our hospital.

\section{Addressing risk factors}

Early identification of risk factors for subsequent infection among CRKP-colonized patients can help in controlling modifiable risk factors (ie, central catheters, urinary catheters, invasive procedures, antibiotic use) and targeting appropriate empirical therapy to minimize morbidity and mortality.

\section{Other interventions}

Selective digestive decontamination of the gastrointestinal tract with oral gentamicin ${ }^{50}$ or a combination of oral gentamicin and colistin ${ }^{51}$ may be used cautiously in a selective patient population, such as transplant recipients or immunocompromised patients colonized with CRKP pending chemotherapy, as well as patients colonized with CRKP who require major intestinal or oropharyngeal surgery.

These interventions have greatly reduced the incidence of colonization and clinical infection with CRKP:

- Ben-David et al used active surveillance during a hospital-wide outbreak of CRKP and showed a 4.7-fold decrease in the incidence of clinical infection, from 6.93 to 1.8 cases per 10,000 patient days. ${ }^{46}$

- Borer et al used a multifaceted strategy during a hospitalwide outbreak of CRKP and reduced the infection density from 5.26 to 0.18 cases per 10,000 patient days. ${ }^{42}$

- Ciobotaro et al used a multidisciplinary intervention to limit the spread of an epidemic CRKP strain and had a 16-fold decrease in CRKP incidence, from 6.6 to 0.5 clinical cases per 10,000 patient days, sustained for 30 months. ${ }^{52}$ Cross-infection decreased from $6.0 \%$ to $2.7 \%$. 
- Schwaber et al and the Israeli CRE Working Group enforced the Israel Ministry of Health guidelines mandating physical separation of hospitalized carriers of CRE and dedicated staffing and appointed a professional task force charged with containment. ${ }^{19}$ The monthly incidence of nosocomial CRE was reduced from 55.5 to 11.7 cases per 100,000 patient days within 15 months.

- Munoz-Price et al used a bundled intervention to control an outbreak of CRKP, successfully preventing horizontal spread of CRKP in a long-term acute care hospital despite ongoing admission of patients colonized with CRKP. ${ }^{49}$

- Kochar et al assessed the effect of enhanced infection control measures with screening for gastrointestinal colonization on limiting the spread of CRKP in a New York hospital endemic for this pathogen. These combined measures were helpful in reducing the incidence of CRKP in an ICU endemic for CRKP from $9.7 \pm 2.2$ to $3.7 \pm 1.6$ new patients per 1,000 patient days per quarter. ${ }^{53}$

CRKP is not limited to acute-care facilities; control of CRKP in long-term care facilities seems to be an essential part to limiting and controlling the spread of this pathogen. Many components of the bundles used in hospitals to combat CRKP could probably be applied successfully in long-term care facilities. Working together on a local and regional level using a multifaceted approach should provide us with better tools to limit the spread of CRKP.

\section{Disclosure}

The authors report no conflicts of interest in this work.

\section{References}

1. Yigit H, Queenan AM, Anderson GJ, et al. Novel carbapenem-hydrolyzing beta-lactamase, KPC-1, from a carbapenem-resistant strain of Klebsiella pneumoniae. Antimicrob Agents Chemother. 2001;45(4): 1151-1161.

2. Naas T, Nordmann P, Vedel G, Poyart C. Plasmid-mediated carbapenemhydrolyzing beta-lactamase KPC in a Klebsiella pneumoniae isolate from France. Antimicrob Agents Chemother. 2005;49(10): 4423-4424.

3. Robustillo Rodela A, Díaz-Agero Pérez C, Sanchez Sagrado T, RuizGarbajosa P, Pita López MJ, Monge V. Emergence and outbreak of carbapenemase-producing KPC-3 Klebsiella pneumoniae in Spain, Sep 2009 to Feb 2010: control measures. Euro Surveill. 2012;17(7):pii:20086.

4. Steinmann J, Kaase M, Gatermann S, et al. Outbreak due to a Klebsiella pneumoniae strain harbouring KPC-2 and VIM-1 in a German university hospital, Jul 2010 to Jan 2011. Euro Surveill. 2011;16(33):pii:19944.

5. Babouee B, Widmer AF, Dubuis O, et al. Emergence of four cases of KPC-2 and KPC-3-carrying Klebsiella pneumoniae introduced to Switzerland, 2009-2010. Euro Surveill. 2011;16(11):pii:19817.

6. Osterblad M, Kirveskari J, Koskela S, et al. First isolations of KPC2-carrying ST258 Klebsiella pneumoniae strains in Finland, Jun and Aug 2009. Euro Surveill. 2009;14(40):pii:19349.

7. Woodford N, Zhang J, Warner M, et al. Arrival of Klebsiella pneumoniae producing KPC carbapenemase in the United Kingdom. J Antimicrob Chemother. 2008;62(6):1261-1264.
8. Giani T, Pini B, Arena F, et al ; AMCLI-CRE Survey Participants. Epidemic diffusion of KPC carbapenemase-producing Klebsiella pneumoniae in Italy: results of the first countrywide survey, May 15, to June 30, 2011. Euro Surveill. 2013;18(22):pii:20489.

9. Samra Z, Ofir O, Lishtzinsky Y, Madar-Shapiro L, Bishara J. Outbreak of carbapenem-resistant Klebsiella pneumoniae producing KPC-3 in a tertiary medical centre in Israel. Int J Antimicrob Agents. 2007;30(6): 525-529.

10. Wiener-Well Y, Rudensky B, Yinnon AM, et al. Carriage rate of carbapenem-resistant Klebsiella pneumoniae in hospitalised patients during a national outbreak. J Hosp Infect. 2010;74(4):344-349.

11. Balkhy HH, El-Saed A, Al Johani SM, et al. The epidemiology of the first described carbapenem-resistant Klebsiella pneumoniae outbreak in a tertiary care hospital in Saudi Arabia: how far do we go? Eur J Clin Microbiol Infect Dis. 2012;31(8):1901-1909.

12. Navon-Venezia S, Leavitt A, Schwaber MJ, et al; Israeli KPC Kpn Study Group. Firstreport on a hyperepidemic clone of KPC-3-producing Klebsiella pneumoniae in Israel genetically related to a strain causing outbreaks in the United States. Antimicrob Agents Chemother. 2009;53(2):818-820.

13. Gregory CJ, Llata E, Stine N, et al. Outbreak of carbapenem-resistant Klebsiella pneumoniae in Puerto Rico associated with a novel carbapenemase variant. Infect Control Hosp Epidemiol. 2010;31(5):476-484.

14. Lopez JA, Correa A, Navon-Venezia S, et al. Intercontinental spread from Israel to Colombia of a KPC-3-producing Klebsiella pneumoniae strain. Clin Microbiol Infect. 2011;17(1):52-56.

15. Roh KH, Lee CK, Sohn JW, Song W, Yong D, Lee K. Isolation of a Klebsiella pneumoniae isolate of sequence type 258 producing KPC-2 carbapenemase in Korea. Korean J Lab Med. 2011;31(4):298-301.

16. Zhang R, Wang XD, Cai JC, et al. Outbreak of Klebsiella pneumoniae carbapenemase 2-producing K. pneumoniae with high qnr prevalence in a Chinese hospital. J Med Microbiol. 2011;60(Pt 7):977-982.

17. Souli M, Galani I, Antoniadou A, et al. An outbreak of infection due to beta-Lactamase Klebsiella pneumoniae Carbapenemase 2-producing K. pneumoniae in a Greek University Hospital: molecular characterization, epidemiology, and outcomes. Clin Infect Dis. 2010;50(3):364-373.

18. Carbonne A, Thiolet JM, Fournier S, et al. Control of a multi-hospital outbreak of KPC-producing Klebsiella pneumoniae type 2 in France, Sep to Oct 2009. Euro Surveill. 2010;15(48):pii:19734.

19. Schwaber MJ, Lev B, Israeli A, et al; Israel Carbapenem-Resistant Enterobacteriaceae Working Group. Containment of a country-wide outbreak of carbapenem-resistant Klebsiella pneumoniae in Israeli hospitals via a nationally implemented intervention. Clin Infect Dis. 2011;52(7):848-855.

20. Chen LF, Anderson DJ, Paterson DL. Overview of the epidemiology and the threat of Klebsiella pneumoniae carbapenemases (KPC) resistance. Infect Drug Resist. 2012;5:133-141.

21. Marchaim D, Chopra T, Pogue JM, et al. Outbreak of colistin-resistant, carbapenem-resistant Klebsiella pneumoniae in metropolitan Detroit, Michigan. Antimicrob Agents Chemother. 2011;55(2):593-599.

22. Shilo S, Assous MV, Lachish T, et al. Risk factors for bacteriuria with carbapenem-resistant Klebsiella pneumoniae and its impact on mortality: a case-control study. Infection. 2013;41(2):503-509.

23. Tzouvelekis LS, Markogiannakis A, Psichogiou M, Tassios PT, Daikos GL. Carbapenemases in Klebsiella pneumoniae and other Enterobacteriaceae: an evolving crisis of global dimensions. Clin Microbiol Rev. 2012;25(4):682-707.

24. Landman D, Babu E, Shah N, et al. Transmission of carbapenemresistant pathogens in New York City hospitals: progress and frustration. J Antimicrob Chemother. 2012;67(6):1427-1431.

25. Prabaker K, Lin MY, McNally M, et al; Centers for Disease Control and Prevention (CDC) Prevention Epicenters Program. Transfer from highacuity long-term care facilities is associated with carriage of Klebsiella pneumoniae carbapenemase-producing Enterobacteriaceae: a multihospital study. Infect Control Hosp Epidemiol. 2012;33(12):1193-1199.

26. Viau RA, Hujer AM, Marshall SH, et al. "Silent" dissemination of Klebsiella pneumoniae isolates bearing K. pneumoniae carbapenemase in a long-term care facility for children and young adults in Northeast Ohio. Clin Infect Dis. 2012;54(9):1314-1321. 
27. Urban C, Bradford PA, Tuckman M, et al. Carbapenem-resistant Escherichia coli harboring Klebsiella pneumoniae carbapenemase beta-lactamases associated with long-term care facilities. Clin Infect Dis. 2008;46(11):e127-e130.

28. Ben-David D, Masarwa S, Navon-Venezia S, et al; Israel PACF CRKP (Post-Acute-Care Facility Carbapenem-Resistant Klebsiella pneumoniae) Working Group. Carbapenem-resistant Klebsiella pneumoniae in post-acute-care facilities in Israel. Infect Control Hosp Epidemiol. 2011;32(9):845-853.

29. Endimiani A, Depasquale JM, Forero S, et al. Emergence of blaKPCcontaining Klebsiella pneumoniae in a long-term acute care hospital: a new challenge to our healthcare system. J Antimicrob Chemother. 2009;64(5):1102-1110.

30. Borer A, Saidel-Odes L, Riesenberg K, et al. Attributable mortality rate for carbapenem-resistant Klebsiella pneumoniae bacteremia. Infect Control Hosp Epidemiol. 2009;30(10):972-976.

31. Schwaber MJ, Klarfeld-Lidji S, Navon-Venezia S, Schwartz D, LeavittA, Carmeli Y. Predictors of carbapenem-resistant Klebsiella pneumoniae acquisition among hospitalized adults and effect of acquisition on mortality. Antimicrob Agents Chemother. 2008;52(3):1028-1033.

32. Mouloudi E, Protonotariou E, Zagorianou A, et al. Bloodstream infections caused by metallo- $\beta$-lactamase/Klebsiella pneumoniae carbapenemase-producing $\mathrm{K}$. pneumoniae among intensive care unit patients in Greece: risk factors for infection and impact of type of resistance on outcomes. Infect Control Hosp Epidemiol. 2010;31(12):1250-1256.

33. Borer A, Saidel-Odes L, Eskira S, et al. Risk factors for developing clinical infection with carbapenem-resistant Klebsiella pneumoniae in hospital patients initially only colonized with carbapenem-resistant K pneumoniae. Am J Infect Control. 2012;40(5):421-425.

34. Kwak YG, Choi SH, Choo EJ, et al. Risk factors for the acquisition of carbapenem-resistant Klebsiella pneumoniae among hospitalized patients. Microb Drug Resist. 2005;11(2):165-169.

35. Wu D, Cai J, Liu J. Risk factors for the acquisition of nosocomial infection with carbapenem-resistant Klebsiella pneumoniae. South Med J. 2011;104(2):106-110.

36. Hussein K, Raz-Pasteur A, Finkelstein R, et al. Impact of carbapenem resistance on the outcome of patients' hospital-acquired bacteraemia caused by Klebsiella pneumoniae. J Hosp Infect. 2013;83(4): 307-313.

37. Schechner V, Kotlovsky T, Kazma M, et al. Asymptomatic rectal carriage of blaKPC producing carbapenem-resistant Enterobacteriaceae: who is prone to become clinically infected? Clin Microbiol Infect. 2013;19(5):451-456.

38. Feldman N, Adler A, Molshatzki N, et al. Gastrointestinal colonization by KPC-producing Klebsiella pneumoniae following hospital discharge: duration of carriage and risk factors for persistent carriage. Clin Microbiol Infect. 2013;19(4):E190-E196.

39. Zimmerman FS, Assous MV, Bdolah-Abram T, Lachish T, Yinnon AM, Wiener-Well Y. Duration of carriage of carbapenem-resistant Enterobacteriaceae following hospital discharge. Am J Infect Control. 2013;41(3):190-194.

40. 2012 CRE Toolkit - Guidance for Control of Carbapenem-resistant Enterobacteriaceae (CRE). Atlanta: Centers for Disease Control and Prevention; 2013. Available from: http://www.cdc.gov/hai/organisms/ cre/cre-toolkit/. Accessed August 15, 2013.

Infection and Drug Resistance

\section{Publish your work in this journal}

Infection and Drug Resistance is an international, peer-reviewed openaccess journal that focuses on the optimal treatment of infection (bacterial, fungal and viral) and the development and institution of preventive strategies to minimize the development and spread of resistance. The journal is specifically concerned with the epidemiology of antibiotic
41. van der Bij AK, Pitout JD. The role of international travel in the worldwide spread of multiresistant Enterobacteriaceae. J Antimicrob Chemother. 2012;67(9):2090-2100.

42. Borer A, Eskira S, Nativ R, et al. A multifaceted intervention strategy for eradication of a hospital-wide outbreak caused by carbapenemresistant Klebsiella pneumoniae in Southern Israel. Infect Control Hosp Epidemiol. 2011;32(12):1158-1165.

43. Grundmann H, Livermore DM, Giske CG, et al; CNSE Working Group. Carbapenem-non-susceptible Enterobacteriaceae in Europe: conclusions from a meeting of national experts. Euro Surveill. 2010;15(46):pii:19711.

44. Munoz-Price LS, Hota B, Stemer A, Weinstein RA. Prevention of bloodstream infections by use of daily chlorhexidine baths for patients at a long-term acute care hospital. Infect Control Hosp Epidemiol. 2009;30(11):1031-1035.

45. Borer A, Gilad J, Porat N, et al. Impact of 4\% chlorhexidine wholebody washing on multidrug-resistant Acinetobacter baumannii skin colonisation among patients in a medical intensive care unit. $J$ Hosp Infect. 2007;67(2):149-155.

46. Ben-David D, Maor Y, Keller N, et al. Potential role of active surveillance in the control of a hospital-wide outbreak of carbapenemresistant Klebsiella pneumoniae infection. Infect Control Hosp Epidemiol. 2010;31(6):620-626.

47. Bilavsky E, Schwaber MJ, Carmeli Y. How to stem the tide of carbapenemase-producing enterobacteriaceae?: proactive versus reactive strategies. Curr Opin Infect Dis. 2010;23(4):327-331.

48. Calfee D, Jenkins SG. Use of active surveillance cultures to detect asymptomatic colonization with carbapenem-resistant Klebsiella pneumoniae in intensive care unit patients. Infect Control Hosp Epidemiol. 2008;29(10):966-968.

49. Munoz-Price LS, Hayden MK, Lolans K, et al. Successful control of an outbreak of Klebsiella pneumoniae carbapenemase-producing $\mathrm{K}$. pneumoniae at a long-term acute care hospital. Infect Control Hosp Epidemiol. 2010;31(4):341-347.

50. Zuckerman T, Benyamini N, Sprecher H, et al. SCT in patients with carbapenem resistant Klebsiella pneumoniae: a single center experience with oral gentamicin for the eradication of carrier state. Bone Marrow Transplant. 2011;46(9):1226-1230.

51. Saidel-Odes L, Polachek H, Peled N, et al. A randomized, doubleblind, placebo-controlled trial of selective digestive decontamination using oral gentamicin and oral polymyxin $\mathrm{E}$ for eradication of carbapenem-resistant Klebsiella pneumoniae carriage. Infect Control Hosp Epidemiol. 2012;33(1):14-19.

52. Ciobotaro P, Oved M, Nadir E, Bardenstein R, Zimhony O. An effective intervention to limit the spread of an epidemic carbapenem-resistant Klebsiella pneumoniae strain in an acute care setting: from theory to practice. Am J Infect Control. 2011;39(8):671-677.

53. Kochar S, Sheard T, Sharma R, et al. Success of an infection control program to reduce the spread of carbapenem-resistant Klebsiella pneumoniae. Infect Control Hosp Epidemiol. 2009;30(5):447-452.

\section{Dovepress}

resistance and the mechanisms of resistance development and diffusion in both hospitals and the community. The manuscript management system is completely online and includes a very quick and fair peerreview system, which is all easy to use. Visit http://www.dovepress.com/ testimonials.php to read real quotes from published authors. 